\title{
Editorial
}

\section{Alternatives Approaches to Treat Biofilm's Infections}

Biofilms, which are the predominant mode of microbial growth, are described as attached and structured microbial communities enclosed by an exopolymeric matrix. Biofilms may be composed of a single-species population or a multiple-species community, but, in both cases, they provide ecologic advantages such as protection from the environment, nutrient availability, metabolic cooperation, and acquisition of new traits. Most probably because of these, biofilms are extremely difficult to eradicate and are a source of many recalcitrant infections. Thus, there is an urgent need to look into and develop new and improved anti-biofilm therapies.

We are very grateful to all the authors for their contribution to this "Special Issue". Three reviews on topics of novelty are included in this issue. They deal with different approaches to treat biofilm's infections span from the oral biofilms, to a novel technique involving a microscale culture of microbial biofilms on a microarray platform, and finally a discussion related to research novel synthetic azoles and natural flavonoids. These reviews, which take into account the medical implications of the treated topics, will be summarized in the following.

The paper by Rodrigues et al. [1] presents an updated review on new approaches in the treatment of oral candidiasis. These included combination anti-Candida therapies, denture cleansers, mouth rinses as alternatives for disrupting candidal biofilms, natural compounds (e.g. honey, probiotics, plant extracts and essential oils) and photodynamic therapy.

Srinivasan et al. [2] alerted to the fact that most of the current techniques for in vitro biofilm formation are not compatible with high throughput screening techniques that can speed up the discovery of new drugs with antibiofilm activity. In order to overcome this problem, the group gave insight into a new method: a microscale culture of microbial biofilms on a microarray platform. The authors show that these nanobiofilms display similar growth, phenotypic and structural properties, permitting a high-throughput screening in search for new anti-biofilm drugs.

Finally, Smiljkovic et al. [3] contributed a current debate on the effects of flavonoids as potential antifungals in Candida albicans infections, discussing the antibiofilm potential and insight into the mode of action. The authors compared the activity of flavonoids with the activity of old and new synthetized azole compounds. This review offered pros and cons for pushing future research regarding exploring novel synthetic azoles and natural flavonoids.

\section{REFERENCES}

[1] Rodrigues, C.F.; Rodrigues, M.E.; Henriques, M. Promising alternative therapeutics for oral candidiasis. Curr. Med. Chem., 2019, 26(14), 2515-2528. DOI: 10.2174/0929867325666180601102333

[2] Srinivasan, A.; Ramasubramanian, A.K.; Lopez-Ribot, J.L. Nano-biofilm arrays as a novel universal platform for microscale microbial culture and high-throughput downstream applications. Curr. Med. Chem., 2019, 26(14), 2529-2535. DOI: $10.2174 / 0929867326666190107155953$

[3] Smiljkovic, M.; Kostic M Stojkovic D, Glamoclija, J.; Sokovic, M. Could flavonoids compete with synthetic azoles in diminishing Candida albicans infections? Curr. Med. Chem., 2019, 26(14), 2536-2554. doi: 10.2174/0929867325666180629133218

\section{Mariana Henriques}

Guest Editor

Laboratory of Research in Biofilms Rosário Oliveira

Centre of Biological Engineering

University of Minho, Braga

Portugal

E-mail: mcrh@deb.uminho.pt;
Célia F. Rodrigues

Guest Editor

Laboratory of Research in Biofilms Rosário Oliveira Centre of Biological Engineering University of Minho, Braga

Portugal and

Laboratory for Process Engineering Environment Biotechnology and Energy-Department of Chemical Engineering,

Faculty of Engineering University of Porto, 4200-465 Porto

Portugal

E-mail: c.fortunae@gmail.com 